\title{
Magnetic Interactions between Nanoparticles Formed during Calcination of Ferrihydrite
}

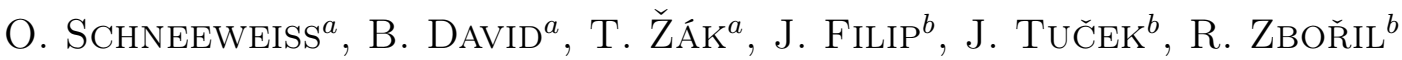 \\ AND M. MAŠLÁ $\check{N}^{b}$ \\ ${ }^{a}$ Institute of Physics of Materials, AS CR, Žižkova 22, 61662 Brno, Czech Republic \\ ${ }^{b}$ Departments of Physical Chemistry and Experimental Physics, Palacky University \\ Tr. 17. listopadu 12, 77146 Olomouc, Czech Republic
}

\begin{abstract}
Magnetic interactions between nanoparticles of magnetite $\mathrm{Fe}_{3} \mathrm{O}_{4}$ and $\alpha$-Fe formed during calcination of ferrihydrite in $\mathrm{H}_{2}$ at temperatures $533 \div 713 \mathrm{~K}$ were studied. The detailed phase analysis describes evolution of contents of magnetite and $\alpha$-Fe. Explanation of an anomalous course of magnetic moment characterized by a local maximum at an early stage of formation of nanoparticles is discussed.
\end{abstract}

PACS numbers: 75.20.Ck, 75.20.En

\section{Introduction}

Thermally induced solid-state conversion of suitable Fe(III) phase under reduction atmosphere has turned out to be one of the most promising approaches leading to a cheap production of iron nanoparticles [1]. The key requirements for suitable Fe-bearing precursor include amorphous or nanocrystalline nature. The main reason is that the structural and phase transformations of amorphous and nanocrystalline materials occur at lower temperatures compared to polycrystalline counterparts [2]. All these requirements are fulfilled in the case of ferrihydrite [3].

In this work, we report on a magnetic interaction which was observed during thermally induced solid-state reduction of natural ferrihydrite in $\mathrm{H}_{2}$ atmosphere in the temperature range of $533-713 \mathrm{~K}$.

\section{Experimental}

The magnetic and phase transformations occurring during thermally induced solid-state reduction of ferrihydrite [4] were derived from isothermal measurements of magnetic moment (ITM) of samples performed in a vibrating sample magnetometer (VSM) in an external field of either 50 Oe or $10 \mathrm{kOe}$ at various temperatures (533-713 K) under $\mathrm{H}_{2}$ atmosphere. Magnetic properties were derived from the hysteresis loops recorded at room temperature in an external field of $\pm 1.5 \mathrm{kOe}$.

The transmission ${ }^{57} \mathrm{Fe}$ Mössbauer spectra were collected using a ${ }^{57} \mathrm{Co}(\mathrm{Rh})$ source. A pure $\alpha$-Fe foil was used as a calibration standard for the velocity scale. The relative contents of individual Fe-bearing phases and their changes were monitored through the corresponding spectral areas. The X-ray powder diffraction (XRD) patterns were measured using a Co $K_{\alpha}$ radiation and analyzed by commercial software and databases.

\section{Results and discussion}

The ITM curves measured at various temperatures (examples are shown in Fig. 1) exhibited a two-step increase of magnetization, except for the lowest temperature $(533 \mathrm{~K})$. The Mössbauer phase analysis of the

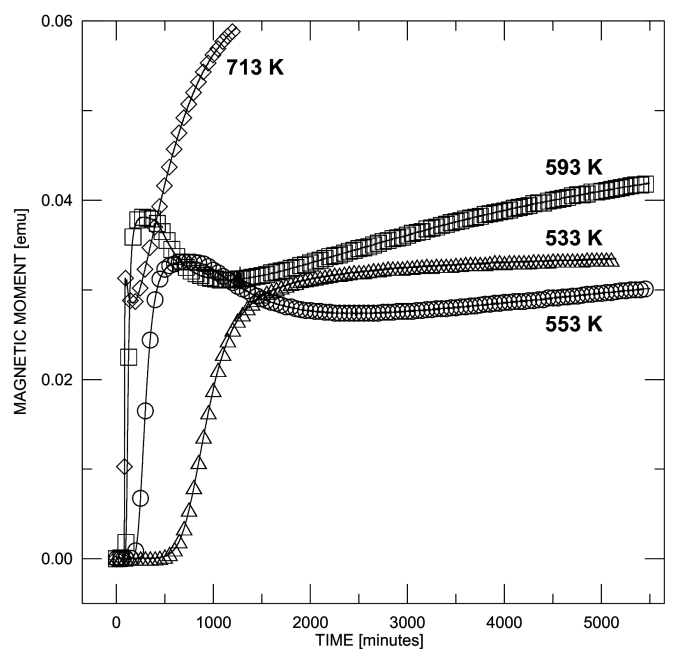

Fig. 1. Time dependences of the magnetic moment of samples annealed in $\mathrm{H}_{2}$ at indicated temperatures in external field of 50 Oe.

samples after ITM measurements taken at temperatures above $533 \mathrm{~K}$ showed magnetite and $\alpha$-Fe phases with a paramagnetic phase represented by the doublet with an isomer shift of $0.35 \mathrm{~mm} / \mathrm{s}$ and quadrupole splitting of $0.69 \mathrm{~mm} / \mathrm{s}$. This phase was interpreted as a non-transformed precursor. In the XRD pattern, peaks of crystalline magnetite and $\alpha$-Fe were identified using PDF. Moreover, two broad peaks were observed and were ascribed to X-ray amorphous-like paramagnetic (XALP) 


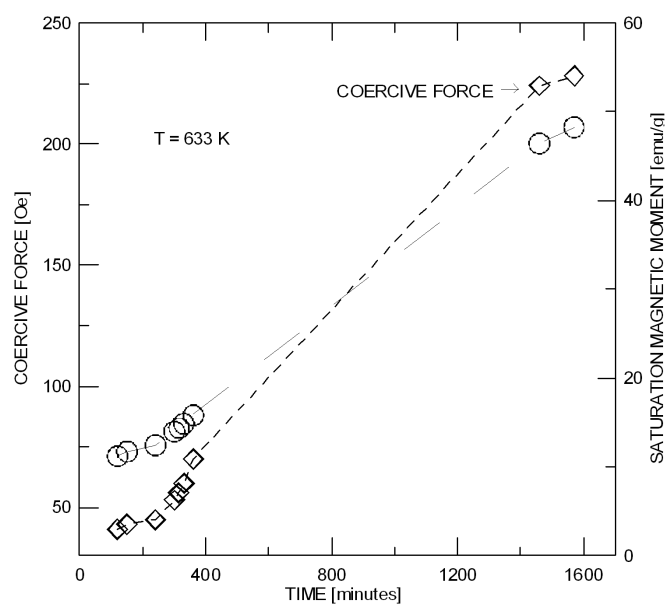

Fig. 2. Time dependence of the coercive force and saturation magnetic moment (at $10 \mathrm{kOe}$ ) of the sample heated at $633 \mathrm{~K}$ in $\mathrm{H}_{2}$.

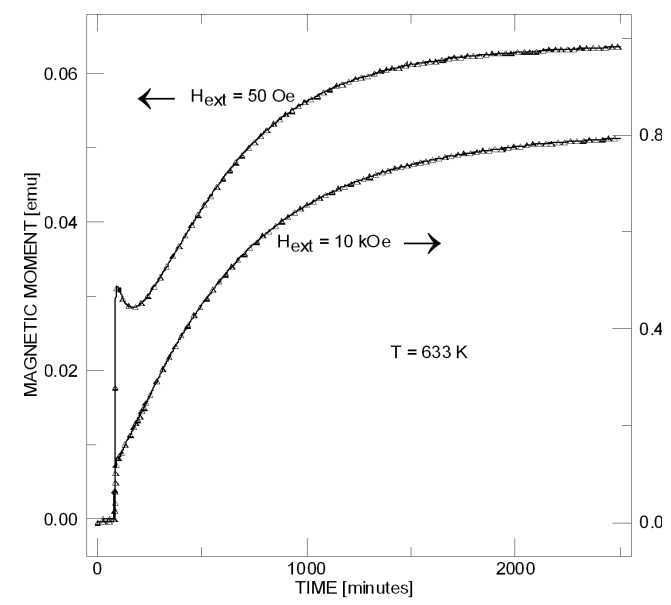

Fig. 3. Time dependence of the magnetic moment of ferrihydrite heated at $633 \mathrm{~K}$ in $\mathrm{H}_{2}$ in external fields of 50 Oe and $10 \mathrm{kOe}$.

phase which corresponded to the paramagnetic phase found in the Mössbauer spectra. The Mössbauer spectrum of the sample after ITM at $533 \mathrm{~K}$ showed the components of magnetite and XALP phase. This result was confirmed by subsequent measurement of the XRD pattern in which crystalline magnetite and XALP phases were identified only. Therefore, the first rapid increase in the magnetic moment (i.e., up to the point where the first maximum in the system magnetic moment appears) was assigned to the growth of magnetite. The first maximum of the magnetic moment is followed by its surprisingly pronounced fall to a local minimum and later by its new increase which is clearly due to the growth of $\alpha$-Fe as confirmed by the Mössbauer and XRD phase analysis. After the fast formation of magnetite $(\approx 14 \%$ of the spectral area at the first maximum), its content remained almost constant for the whole time of annealing. The content of $\alpha$-Fe phase grew at the expense of XALP. This is con- nected with a raise in the coercive force and saturation moment (see Fig. 2). Hence, the anomalous fall in the magnetic moment at the initial stage of $\alpha$-Fe formation needs to be explained.

In order to prove a possible antiparallel orientation of the moments in magnetite vs. $\alpha$-Fe nanoparticles, ITM measurement in a field of $10 \mathrm{kOe}$ was carried out. The local minimum on the ITM curve was not observed in this case (see Fig. 3).

The hysteresis loops measured on the samples prepared by ITM measurements at $633 \mathrm{~K}$ for $2400 \mathrm{~min}$ in external fields of 50 Oe and $10 \mathrm{kOe}$ exhibited the same values of the coercive force $(310 \pm 10 \mathrm{Oe})$ but the approach to saturation in fields below $9 \mathrm{kOe}$ is slower for the sample annealed in 50 Oe which is in agreement with an antiparallel orientation of the moments. Therefore, the different orientation of magnetic moments of magnetite and $\alpha$-Fe nanoparticles, related to the different crystal growth of both phases, is the only response to the strength of the external magnetic field. An antiparallel orientation of the particle moments of the different phases can be simply ascribed to a tendency of the particle system to minimize its stray fields and energy.

\section{Conclusion}

A nanocrystalline/amorphous ferrihydrite was converted by a thermal treatment in $\mathrm{H}_{2}$ atmosphere into magnetite and bcc $\alpha$-Fe nanoparticles with various contents of the remaining XALP phase. The local minimum on the ITM curves can be explained as an antiparallel orientation of primarily formed magnetite to the later growing $\alpha$-Fe nanoparticles. The different orientation of the magnetic moments of magnetite and $\alpha$-Fe nanoparticles can be overcome by heating the sample in a stronger external field $(10 \mathrm{kOe})$, which induces the preferential growth of both phases with their moments oriented more parallel with respect to the external field.

\section{Acknowledgments}

This work has been supported by the projects of the Ministry of Education of the Czech Republic (1M619895201) and Grant Agency of the Czech Republic $(106 / 08 / 1440)$.

\section{References}

[1] R. Zboril, M. Mashlan, D. Petridis, Chem. Mater. 14, 969 (2002).

[2] D.V. Talapin, E.V. Shevchenko, H. Weller, in: Nanoparticles, From Theory to Application, Ed. G. Schmid, Wiley, Berlin 2003, p. 199.

[3] R.M. Cornell, U. Schwertmann, The Iron Oxides, Wiley-VCH, Weinheim 2003.

[4] J. Filip, R. Zboril, O. Schneeweiss, J. Zeman, M. Cernik, P. Kvapil, M. Otyepka, Environ. Sci. Technol. 41, 4367 (2007). 